\title{
Efficacy of Combining Proximal Balloon Guiding Catheter and Distal Access Catheter in Thrombectomy with Stent Retriever for Anterior Circulation Ischemic Stroke
}

\author{
Sang Hwa Kim, ${ }^{1,2}$ Jae Hyung Choi ${ }^{1,2}$ Myung Jin Kang, ${ }^{1,3}$ Jae Kwan Cha, ${ }^{1,4}$ Dae Hyun Kim, ${ }^{1,4}$ Hyun Wook Nah, ${ }^{1,4}$ Hyun Seok Park, ${ }^{1,2}$ \\ Sang Hyun Kim, ${ }^{1,3}$ Jae Taeck Huh ${ }^{1,2}$ \\ Busan-Ulsan Regional Cardiocerebrovascular Center, Dong-A University Hospital, Busan, Korea \\ Department of Neurosurgery, ${ }^{2}$ Dong-A University Hospital, Dong-A University College of Medicine, Busan, Korea \\ Department of Radiology, ${ }^{3}$ Dong-A University Hospital, Dong-A University College of Medicine, Busan, Korea \\ Department of Neurology, ${ }^{4}$ Dong-A University Hospital, Dong-A University College of Medicine, Busan, Korea
}

Objective : We evaluated efficacy of combining proximal balloon guiding catheter (antegrade flow arrest) and distal access catheter (aspiration at the site of occlusion) in thrombectomy for anterior circulation ischemic stroke.

Methods : We retrospectively analyzed 116 patients who underwent mechanical thrombectomy with stent retriever. The patients were divided by the techniques adopted, the combined technique (proximal balloon guiding catheter and large bore distal access catheter) group ( $n=57,49.1 \%$ ) and the conventional (guiding catheter with stent retriever) technique group ( $n=59,50.9 \%)$. We evaluated baseline characteristics (epidemiologic data, clinical and imaging characteristics) and procedure details (the number of retrieval attempts, procedure time), as well as angiographic (thrombolysis in cerebral infarction (TICI) score, distal thrombus migration) and clinical outcome (National Institutes of Health Stroke Scale at discharge, modified Rankin Scale [mRS] at 3 months) of them.

Results : The number of retrieval attempts was lower $(p=0.002)$ and the first-pass successful reperfusion rate was higher $(56.1 \%$ vs. $28.8 \% ; p=0.003)$ in the combined technique group. And the rate of final result of $\mathrm{TICl}$ score 3 was higher $(68.4 \% \mathrm{vs.} 28.8 \% ; p<0.01)$ and distal thrombus migration rate was also lower $(15.8 \%$ vs. $40.7 \% ; p=0.021)$ in the combined technique group. Early strong neurologic improvement (improvement of National Institutes of Health Stroke Scale $\geq 11$ or National Institutes of Health Stroke Scale $\leq 1$ at discharge) rate $(57.9 \%$ vs. $36.2 \% ; p=0.02)$ and favorable clinical outcome (mRS at 3 months $\leq 2)$ rate $(59.6 \%$ vs. $33.9 \% ; p=0.005$ ) were also better in the combined technique group.

Conclusion : The combined technique needs lesser attempts, decreases distal migration, increases $\mathrm{TICl} 3$ reperfusion and achieves better clinical outcomes.

Key Words : Stroke · Brain ischemia $\cdot$ Thrombectomy $\cdot$ Stents.

- Received : January 3, $2019 \bullet$ Revised : March 30, $2019 \bullet$ Accepted : April 24, 2019

- Address for reprints : Jae Hyung Choi

Department of Neurosurgery, Busan-Ulsan Regional Cardiocerebrovascular Center, Dong-A University Hospital, Dong-A University College of Medicine, 32 Daesingongwonro, Seo-gu, Busan 49202, Korea

Tel : +82-51-240-5241, Fax : +82-51-242-6714, E-mail : nschoi1018@dau.ac.kr, ORCID : https://orcid.org/0000-0003-1173-2787

This is an Open Access article distributed under the terms of the Creative Commons Attribution Non-Commercial License (http://creativecommons.org/licenses/by-nc/4.0) which permits unrestricted non-commercial use, distribution, and reproduction in any medium, provided the original work is properly cited. 


\section{INTRODUCTION}

Proximal flow control with continuous aspiration using balloon-guiding catheter (BGC) can reduce the number of retrieval attempt and the risk of distal embolization and can also increase the recanalization rate on hard and large burden $\operatorname{clot}^{6,15)}$. And theoretically, the use of large bore distal access catheter (DAC) may have some advantages on reducing the distance of the thrombus float in the vessels and aspirating the thrombus directly. Actually, there has been several in vitro studies, which reported additional proximal control and/or distal aspiration during mechanical thrombectomy can improve recanalization rate and reduce distal embolization ${ }^{1,2)}$.

So, we hypothesized that the combination of the two techniques (antegrade flow arrest with BGC and aspiration with large bore DAC at the site of occlusion) can improve recanalization success rate and clinical outcomes, and procedural efficiency in stent retriever assisted mechanical thrombectomy.

We compared angiographic and clinical outcome data of the patients who received combined (BGC+DAC) technique with that of the patients who received conventional guiding catheter with stent retriever assisted mechanical thrombectomy.

\section{MATERIALS AND METHODS}

\section{Data collection}

We retrospectively reviewed clinical and imaging data of 116 patients treated with mechanical thrombectomy for acute ischemic stroke at our institution, between March 2014 and December 2017. Fifty-nine patients received conventional technique and 57 patients received combined technique. The raw data were obtained from stroke registry data collected prospectively. All patients with anterior circulation occlusion such as Middle Cerebral Artery (MCA) occlusion and distal Internal Carotid Artery (ICA) occlusion were enrolled. Cerebral infarction caused by distal common carotid artery, proximal ICA, and posterior circulation were excluded for unified comparison of clinical and radiological results. Our Institutional Review Board approved this study for retrospective analysis.

\section{Procedure}

Conventional technique; guiding catheter with stent retriever

Procedures were performed with a $90 \mathrm{~cm} 6$ French (Fr) Envoy guiding catheter (Cordis, Miami Lakes, FL, USA) placed in the petrous or cavernous segment of ICA. Control angiograms were performed to verify arterial occlusion sites and determine the collateral status. After verification of the arterial occlusion, the ICA and occluded MCA segment were accessed with a 0.014 -inch microwire and a microcatheter $(0.018$ or 0.027 inch) capable of delivering a $4 \mathrm{~mm}$ or $6 \mathrm{~mm}$ diameter standard stent retriever. After placement of microcatheter distal to the thrombus, the stent retriever was deployed and a control angiogram was performed to determine the immediate reperfusion status. The stent retrievers used in this study were Solitaire (Medtronic Neurovascular, Irvine, CA, USA), and Trevo (Concentric Medical, Mountain View, CA, USA) devices. Stent was left deployed for 3-5 minutes, and then, the stent retriever and microcatheter were retracted together with continuous aspiration using a $50 \mathrm{~mL}$ syringe connected to the guiding catheter.

\section{Combined technique; proximal BGC+large bore DAC}

On this technique, proximal occlusion and distal aspiration were performed simultaneously. After groin puncture of the femoral artery with 8 Fr introducer sheath, 8 Fr BGC (Cello balloon guide catheter; Covidien, Irvine, CA, USA) was positioned in the ICA cervical segment of the affected side. Subsequently, the large bore DAC (5 Fr Navien distal access catheter; Medtronic Neurovascular) was advanced through the BGC and navigated as close to the thrombus as possible. After positioning a microcatheter distal to the thrombus, the stent retriever was deployed in the same way with the conventional technique (Fig. 1A and B).

The balloon was inflated just before stent retriever retraction and vacuum was applied in the large bore DAC with a 50 $\mathrm{mL}$ syringe (Fig. 1C). Under the negative pressure through the large bore DAC, the deployed stent retriever was pulled back carefully into the large bore DAC and pulled out into the hemostatic valve. After retracting the stent retriever, if the free aspiration was possible through the large bore DAC, the large bore DAC was left in place. Contrary, if the clot was apparently stuck in the catheter, it was also removed through BGC un- 

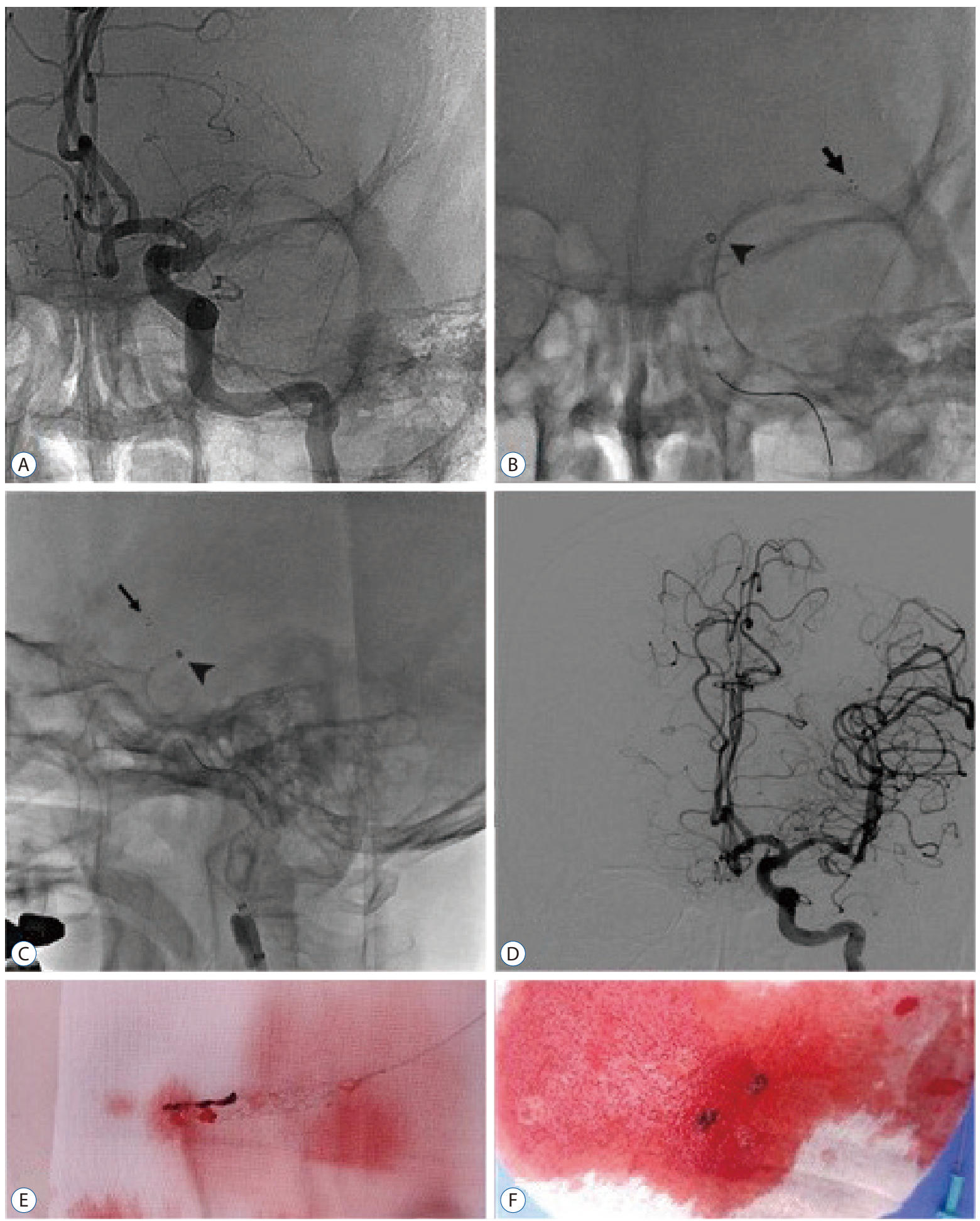

Fig. 1. Angiograms of "the combined technique" for left proximal M1 occlusion. A : Pre-treatment angiogram (antero-posterior view) shows the occlusion of left proximal M1 segment. B : A Solitaire stent retriever was deployed within the left M1 segment. Arrow indicates the distal marker of Solitaire stent. Arrowhead points the location of the large bore distal access catheter for aspiration. $C$ : The balloon catheter was inflated for proximal flow arrest (lateral view). Arrow indicates the distal marker of Solitaire stent. Arrowhead indicates the location of the large bore distal access catheter for aspiration. D : After first retrieval under simultaneous distal aspiration with proximal flow arrest, angiogram shows complete recanalization without distal thrombus migration (thrombolysis in cerebral infarction 3). E and F : Photographs shows retrieved clot fragment within Solitaire stent retriever (E) and aspirated clot within the distal access catheter (F). 
der continuous aspiration (Fig. 1D-F).

\section{Radiological assessment}

To specify recanalization success, the degree of post-intervention vessel occlusion was classified by Thrombolysis In Cerebral Infarction (TICI) score and the final result of TICI $2 \mathrm{~b}$ or 3 was defined as successful recanalization ${ }^{7)}$.

Procedure related adverse events were also noted, such as distal thrombus migration and hemorrhagic complications. Distal thrombus migration (defined as visible thrombus fragment or emboli which occlude distal segment of the primary site or other vascular territory on angiography) was assessed strictly by comparing angiographic images which obtained at the start and the end of the procedure. Procedure related hemorrhage was assessed by follow-up computed tomography images performed within 24 hours after procedure. Symptomatic hemorrhage was defined as any intracranial hemorrhage (parenchymal hematoma, subarachnoid hemorrhage, or intraventricular hemorrhage) associated with a worsening of $\mathrm{Na}-$ tional Institutes of Health Stroke Scale (NIHSS) score by $\geq 4$ within 24 hours. The time interval from symptom onset to femoral artery puncture time and the procedure time (defined as the time from the femoral artery puncture to the end of recanalization) were recorded.

\section{Clinical assessment}

Clinical outcome was measured by post procedural NIHSS score assessed at discharge by qualified neurologist and modified Rankin Scale (mRS) at 3 months after discharge. "Strong neurologic improvement" was defined as improvement of NIHSS score by $\geq 11$ or NIHSS score at discharge $\leq 1$. This criterion was chosen based on previous analysis using NIHSS-based endpoint ${ }^{9,18)}$.

A mRS score at 3 months after discharge was obtained during routine clinical follow-up visits performed by trained research nurses unware of the study group assignments. For the patients unable to visit our institution for follow-up, mRS scores were obtained via telephone conversation with the patients, their relatives, or general practitioners. A favorable clinical outcome was defined as functional independence, mRS score $0,1,2$ at the time of 3 months follow-up.

\section{Statistical analysis}

Baseline characteristics and procedure details, as well as angiographic and clinical outcome were compared between two groups. Comparison between them was performed using Fisher's exact test, chi-square tests, Wilcoxon rank sum test and independent $\mathrm{t}$ tests (significant level 0.05). All statistical analyses were performed using JMP version 10 (SAS Institute, Inc, Cary, NC, USA).

Table 1. Baseline characteristics of the combined technique group and the conventional technique group

\begin{tabular}{|c|c|c|c|c|}
\hline & Total $(n=116)^{*}$ & Combined $(n=57)^{*}$ & Conventional $(n=59)^{*}$ & $p$-value \\
\hline Sex (male : female) & $57: 59$ & $26: 31$ & $31: 28$ & 0.456 \\
\hline Age (years) & $67.61 \pm 10.36$ & $69.77 \pm 10.20$ & $65.53 \pm 10.16$ & 0.027 \\
\hline Vessel (MCA : distal ICA) & $71: 45$ & $30: 27$ & $41: 18$ & 0.062 \\
\hline Hypertension (no : yes) & $61: 55$ & $24: 33$ & $37: 22$ & 0.026 \\
\hline Diabetes mellitus (no : yes) & $96: 20$ & $47: 10$ & $49: 10$ & 0.932 \\
\hline Smoking (no: yes) & $86: 30$ & $40: 17$ & $46: 13$ & 0.371 \\
\hline Hyperlipidemia (no : yes) & $106: 10$ & $50: 7$ & $56: 3$ & 0.167 \\
\hline Atrial fibrillation (no : yes) & $47: 69$ & $21: 36$ & $26: 33$ & 0.428 \\
\hline Coronary artery disease (no : yes) & $99: 17$ & $50: 7$ & $49: 10$ & 0.477 \\
\hline Old cerebrovascular accident (no : yes) & $94: 22$ & $46: 11$ & $48: 11$ & 0.928 \\
\hline IV tPA (no : yes) & $56: 60$ & $31: 26$ & $25: 34$ & 0.195 \\
\hline Initial NIHSS (score) & $13.79 \pm 4.16$ & $13.37 \pm 4.42$ & $14.20 \pm 3.88$ & 0.281 \\
\hline Onset to puncture time (minutes) & $248.95 \pm 165.64$ & $255.32 \pm 213.81$ & $242.69 \pm 99.39$ & 0.685 \\
\hline
\end{tabular}

Values are presented as mean \pm standard deviation unless otherwise indicated. *Number of patients. MCA : middle cerebral artery, ICA : internal carotid artery, IV tPA : intravenous tissue plasminogen activator, NIHSS : National Institutes of Health Stroke Scale 


\section{RESULTS}

A total of 116 patients underwent mechanical thrombectomy using a stent retriever. Of the 116 patients, 57 patients (49.1\%) were treated by the combined technique. Mean age was 67.6 years, and 59 of the patients (50.9\%) were women. Vessel occlusion was located in MCA (M1 and M2) segment in 71 cases $(61.2 \%)$, in distal ICA in 45 cases (38.8\%). The baseline characteristics for age, sex, medical histories, initial
NIHSS score and symptom onset to femoral artery puncture time in the combined technique group and the conventional technique group were largely comparable (Table 1).

In radiologic outcomes, rate of successful reperfusion was higher (TICI grade $2 \mathrm{~b}$ or 3 ) in the combined technique group than in the conventional technique group but did not show statistical significance ( $86 \%$ vs. $71.2 \% ; p=0.053$ ). However, the rate of TICI 3 reperfusion of the combined technique group was significantly higher than that of the conventional tech-

Table 2. Radiologic outcomes of the combined technique group and the conventional group

\begin{tabular}{|c|c|c|c|c|}
\hline & Total $(n=116)^{*}$ & Combined $(n=57)^{*}$ & Conventional $(\mathrm{N}=59)^{*}$ & $p$-value \\
\hline \multicolumn{5}{|c|}{$\mathrm{TICl}$ grade } \\
\hline 0 & $17(14.7)$ & $4(7.0)$ & $13(22.0)$ & $<0.001$ \\
\hline 1 & $4(3.4)$ & $2(3.5)$ & $2(3.4)$ & \\
\hline $2 a$ & $4(3.4)$ & $2(3.5)$ & $2(3.4)$ & \\
\hline $2 b$ & $35(30.2)$ & $10(17.5)$ & $25(42.4)$ & \\
\hline 3 & $56(48.3)$ & $39(68.4)$ & $17(28.8)$ & $<0.001$ \\
\hline $2 b+3$ & $91(78.4)$ & $49(86.0)$ & $42(71.2)$ & 0.053 \\
\hline \multicolumn{5}{|c|}{ Migration } \\
\hline 0 & $83(71.6)$ & $48(84.2)$ & $35(59.3)$ & 0.021 \\
\hline 1 & $21(18.1)$ & $5(8.8)$ & $16(27.1)$ & \\
\hline 2 & $5(4.3)$ & $1(1.8)$ & $4(6.8)$ & \\
\hline 3 & $7(6.0)$ & $3(5.3)$ & $4(6.8)$ & \\
\hline 4 & $0(0.0)$ & $0(0.0)$ & $0(0.0)$ & \\
\hline
\end{tabular}

Values are presented as number (\% or ratio). *Number of the patients. $\mathrm{TICl}$ : thrombolysis in cerebral infarction, Migration : distal migration, 1 : distal migration to M3 segment, 2 : distal migration to anterior cerebral artery, 3 : distal migration to both anterior cerebrl artery and middle cerebral artery, 4 : distal migration to posterior cerebral artery

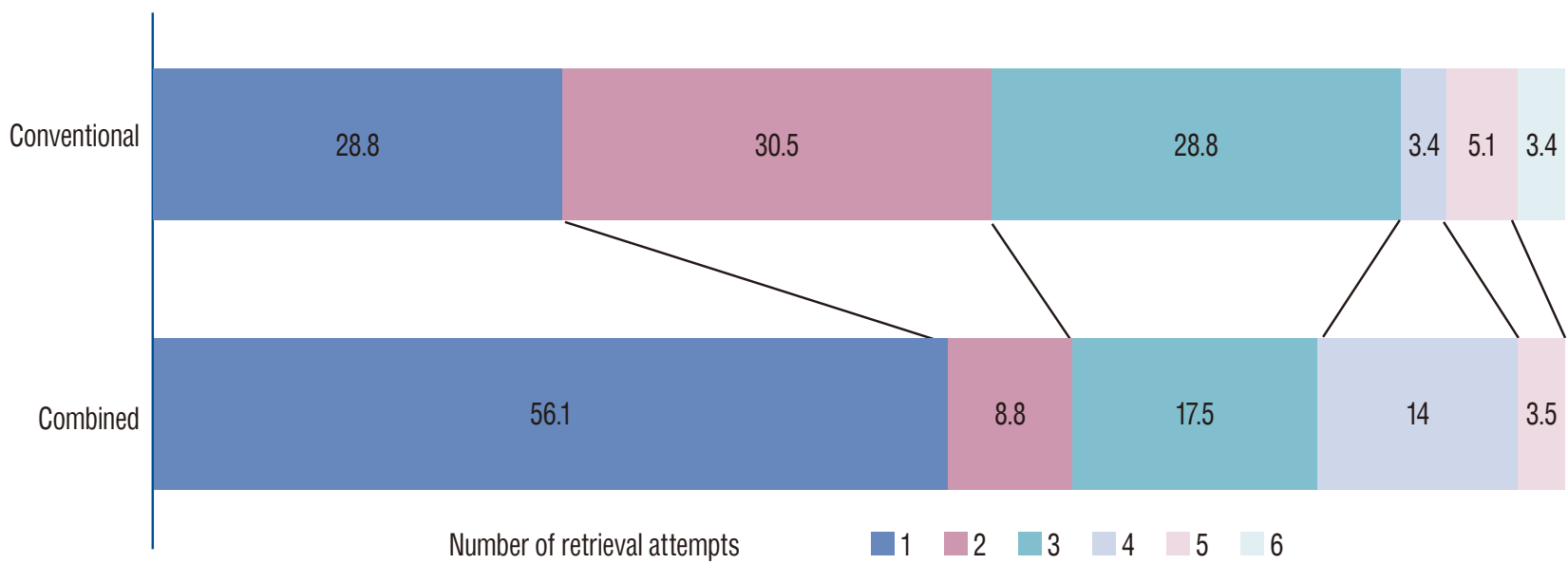

Fig. 2. Distribution of the number of retrieval attempts in the combined technique group and the conventional technique group. The percentage of patients is shown in each cell. 
nique group (68.4\% vs. $28.8 \%$; $p<0.001)$. Distal thrombus migration was usually observed in M3 segment (18.1\%) than other new vascular territory (10.3\%) and the rate of distal thrombus migration was significantly lower in the combined technique group (15.8\% vs. $40.7 \%$; $p=0.021$ ) (Table 2).
The mean procedure time was shorter in the combined technique group but statistically insignificant $(56.26 \pm 25.72$ minutes vs. $64.17 \pm 31.72$ minutes; $p=0.145$ ). Procedure related intracranial hemorrhage rate was much lower in the combined technique group $(12.2 \%$ vs. $45.8 \% ; p<0.001)$, but the rate of

Table 3. Procedural results of the combined technique group and the conventional group

\begin{tabular}{|c|c|c|c|c|}
\hline & Total $(n=116)^{*}$ & Combined $(n=57)^{*}$ & Conventional $(n=59)^{*}$ & $p$-value \\
\hline \multicolumn{5}{|l|}{ Number of retrieval attempts } \\
\hline 1 & $49(42.2)$ & $32(56.1)$ & $17(28.8)$ & 0.002 \\
\hline 2 & $23(19.8)$ & $5(8.8)$ & $18(30.5)$ & \\
\hline 3 & $27(23.3)$ & $10(17.5)$ & $17(28.8)$ & \\
\hline 4 & $10(8.6)$ & $8(14.0)$ & $2(3.4)$ & \\
\hline 5 & $5(4.3)$ & $2(3.5)$ & $3(5.1)$ & \\
\hline 6 & $2(1.7)$ & $0(0.0)$ & $2(3.4)$ & \\
\hline 1 & $49(42.2)$ & $32(56.1)$ & $17(28.8)$ & 0.003 \\
\hline$\geq 2$ & $67(57.8)$ & $25(43.9)$ & $42(71.2)$ & \\
\hline Procedure related ICH & $34(29.3)$ & $7(12.2)$ & $27(45.8)$ & $<0.001$ \\
\hline Symptomatic ICH & $12(10.3)$ & $5(8.8)$ & $7(11.9)$ & 0.585 \\
\hline Procedure time (minutes) & $60.25 \pm 29.05$ & $56.26 \pm 25.72$ & $64.17 \pm 31.72$ & 0.145 \\
\hline $\begin{array}{l}\text { Procedure time of } \mathrm{TICI} 2 \mathrm{~b}+3(\mathrm{n}=91)^{*} \\
\text { reperfusion (minutes) }\end{array}$ & $52.67 \pm 24.69$ & $51.35 \pm 23.60$ & $54.21 \pm 26.10$ & 0.584 \\
\hline
\end{tabular}

Values are presented as mean \pm standard deviation or number (\%). *Number of patients. ICH : intracranial hemorrhage, $\mathrm{TICl}$ : thrombolysis in cerebral infarction

Table 4. Clinical outcomes of the combined technique group and the conventional group

\begin{tabular}{|c|c|c|c|c|}
\hline & Total $(n=116)^{*}$ & Combined $(n=57) *$ & Conventional $(n=59)^{*}$ & $p$-value \\
\hline Initial NIHSS (score) & $13.79 \pm 4.16$ & $13.37 \pm 4.42$ & $14.20 \pm 3.88$ & 0.281 \\
\hline NIHSS at discharge (score) & $6.00 \pm 5.70$ & $5.32 \pm 5.62$ & $6.73 \pm 5.76$ & 0.212 \\
\hline Improvement of $\mathrm{NIHSS} \geq 11$ or $\mathrm{NIHSS} \leq 1$ (no: yes) & $61(53.0): 54(47.0)$ & $24(42.1): 33(57.9)$ & $37(63.8): 21(36.2)$ & 0.020 \\
\hline \multicolumn{5}{|l|}{$\mathrm{mRS}$ at 3 months after discharge } \\
\hline 0 & $9(7.8)$ & $5(8.8)$ & $4(6.8)$ & 0.130 \\
\hline 1 & $24(20.7)$ & $17(29.8)$ & $7(11.9)$ & \\
\hline 2 & $21(18.1)$ & $12(21.1)$ & $9(15.3)$ & \\
\hline 3 & $18(15.5)$ & $8(14.0)$ & $10(16.9)$ & \\
\hline 4 & $20(17.2)$ & $6(10.5)$ & $14(23.7)$ & \\
\hline 5 & $9(7.8)$ & $4(7.0)$ & $5(8.5)$ & \\
\hline 6 & $15(12.9)$ & $5(8.8)$ & $10(16.9)$ & \\
\hline \multicolumn{5}{|l|}{ Favorable functional outcome } \\
\hline$\leq 2$ & $54(46.6)$ & $34(59.6)$ & $20(33.9)$ & 0.005 \\
\hline$\geq 3$ & $62(53.4)$ & $23(40.4)$ & $39(66.1)$ & \\
\hline
\end{tabular}

Values are presented as mean \pm standard deviation or number (\%) unless otherwise indicated. *Number of patients. NIHSS : National Institutes of Health Stroke Scale, mRS : modified Rankin Scale 
symptomatic hemorrhage was similar in both groups $(8.8 \%$ vs. $11.9 \%$; $p=0.585)$. The number of retrieval attempts was lower in the combined technique group ( $p=0.002$ ) (Fig. 2) and especially, the first-pass successful reperfusion rate was significantly higher in the combined technique group $(56.1 \%$ vs. $28.8 \%$; $p=0.003$ ) (Table 3).

On the side of clinical outcomes, patients in the combined technique group showed substantially better results. NIHSS scores at discharge were lower in the combined technique group but did not show statistically significant difference. However, the combined technique group had higher rates of strong neurologic improvement $(57.9 \%$ vs. $36.2 \% ; p=0.02)$ and higher rates of favorable functional outcome at 3 months (59.6\% vs. $33.9 \%$; $p=0.005)$ compared with that of the conventional technique group (Table 4, Fig. 3).

\section{DISCUSSION}

The technology of neuroendovascular devices has been improved considerably. In addition to improvements of mechanical thrombectomy devices, adjunctive catheters such as balloon guiding catheter or distal access catheter also achieved a remarkable development. In recent, different adjunctive technical approaches, such as proximal flow arrest using BGC or distal aspiration with large bore DAC, are combined with stent retriever ${ }^{4,5,12,14,17)}$.

After recent several randomized controlled trials, mechanical thrombectomy using stent retriever is now considered the standard of care in the patients with acute ischemic stroke due to large vessel occlusion ${ }^{16}$. The method of mechanical thrombectomy has continued to evolve and improve. According to a recent meta-analysis of individual data about endovascular therapy, successful recanalization is highly achieved in $71 \%$ of cases with $46 \%$ of independent functional outcomes ${ }^{5}$. However, there still remains a sizable minority of patients refractory to conventional mechanical thrombectomy and there also remains discrepancy between recanalization rates and clinical outcome rates.

Our concept of the combined technique is based on the idea of combining two adjunctive techniques (proximal balloon occlusion and distal aspiration) on mechanical thrombectomy using stent retriever. The results of our study comparing the combined technique group with the conventional group revealed lower distal migration rates and better clinical outcomes of patients treated with the combined technique. And the rate of successful recanalization in the combined technique group were $86 \%$. These results were better than metaanalysis of five randomized controlled trial and matching the highest rate reported in the literature (the HERMES analysis and the STRATIS registry) $)^{5,13)}$.

There are several in vitro and in vivo studies about the use of BGC or DAC in conjunction with stent retriever ${ }^{1,2,8,15}$. They demonstrated that the application of BGC for proximal flow arrest was the best way to prevent fragmentation of soft elastic clots and aspiration using distal access catheter was the best way to reduce hard clot fragmentation ${ }^{1,3)}$. In addition, distal aspiration by DAC may increase suction effect by reducing de-

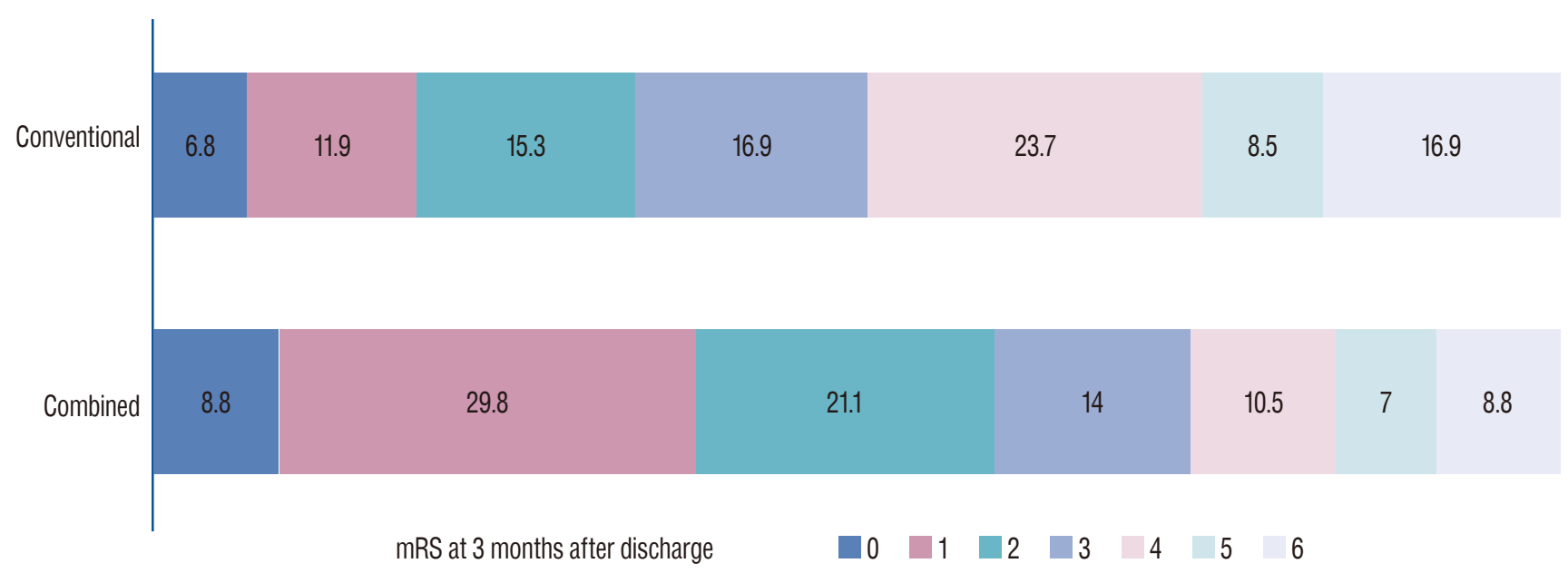

Fig. 3. Distribution of the $\mathrm{mRS}$ at 3 months in the combined technique group and the conventional technique group. The percentage of patients is shown in each cell. $\mathrm{mRS}$ : modified Rankin Scale. 
layed flow restoration via ophthalmic, anterior cerebral, and posterior communicating arteries, which can decrease aspiration effect in BGC only method. And the distance of the thrombus float in the vessels can be minimized by distal aspiration. In this way, mechanical extraction effect can be improved. So, theoretically, a multimodal approach using all of these tools can increase successful recanalization rates and lower distal thrombus migration rates.

As described in this study, the combined technique group showed lesser retrieval attempts and significantly higher rate of first-pass recanalization. And the rates of distal thrombus migration rate were significantly lower in the combined technique group and therefore higher complete (TICI 3) reperfusion rate was achievable. We suggest that higher rate of TICI 3 reperfusion also attributed better clinical outcome in the combined technique group. There are several studies shown that the patients with complete reperfusion (TICI 3) had a better functional outcome than those who achieved TICI $2 \mathrm{~b}$ reperfusion. Those studies demonstrated that the patients with TICI 3 reperfusion had not only lower NIHSS scores but also higher rates of independent functional outcome ( $\mathrm{mRS} 0$, 1) at 3 months ${ }^{10,11)}$. In our study, difference of NIHSS score at discharge between two groups was not statistically significant. But strong neurologic improvement rate and favorable functional outcome rate were significantly higher in the combined technique group.

We also suggest higher first-pass recanalization rate may contributed to lesser procedure related intracranial hemorrhage in the combined technique group $(12.2 \%$ vs. $45.8 \%$; $p<0.001$ ), even though the rate of symptomatic hemorrhage was similar in both groups.

One of the concerns with using the combined technique is a higher complexity of the procedure which can increase procedure time. However, in our study, even if there was no statistical significance, the combined technique group had shorter procedure time. In our opinion, the reason of shorter procedure time was considerably related with lesser retrieval attempts.

\section{Limitation}

The patient's data of this study were analyzed retrospectively from the single institution with modest sample size, and there was lack of external adjudication of angiographic findings and clinical outcomes. In addition, patient populations in the two groups were somewhat heterogenous (including three different operators, the operator's learning curve over the periods, a higher proportion of distal ICA occlusion and relatively high mean age in the conventional technique group). And the stent retrievers used in this study were also heterogenous. This study design couldn't identify which portion of the combined technique (flow arrest by BGC or direct aspiration by DAC) had more contributed to better outcomes. In order to drive more definite conclusions about this technique, welldesigned prospective trials are necessary to define optimal therapeutic strategies for the patients with acute ischemic stroke.

\section{CONCLUSION}

The combined technique, using balloon guiding catheter and distal access catheter simultaneously, improved first-pass successful reperfusion rate and reduced distal thrombus migration. And the combined technique also achieved higher rate of complete reperfusion (TICI 3) and better clinical outcomes even in the cases of larger thrombus load. We recommend the use of the combined technique during mechanical thrombectomy in patients with anterior circulation ischemic stroke.

\section{CONFLICTS OF INTEREST}

No potential conflict of interest relevant to this article was reported.

\section{INFORMED CONSENT}

This type of study does not require informed consent.

\section{AUTHOR CONTRIBUTIONS}

\author{
Conceptualization : SHK, JHC, MJK, JKC \\ Data curation : SHK, JHC, MJK, JKC, DHK, HWN, HSP, \\ SHK, JTH
}

Formal analysis : SHK, JHC 
Methodology : SHK, JHC, MJK, JKC

Project administration : SHK, JHC, MJK, JKC

Visualization : SHK, JHC, MJK, SHK

Writing - original draft : SHK, JHC

Writing - review \& editing : SHK, JHC, MJK, JKC, DHK, HWN, HSP, SHK, JTH

\section{References}

1. Chueh JY, Kühn AL, Puri AS, Wilson SD, Wakhloo AK, Gounis MJ : Reduction in distal emboli with proximal flow control during mechanical thrombectomy: a quantitative in vitro study. Stroke 44 : 1396-1401, 2013

2. Chueh JY, Puri AS, Wakhloo AK, Gounis MJ : Aspiration efficacy of suction catheter in the management of distal embolization during interventional treatment of acute ischemic stroke. J Neurointerv Surg 6 (Suppl 1) : A1-A78, 2014

3. Chueh JY, Puri AS, Wakhloo AK, Gounis MJ : Risk of distal embolization with stent retriever thrombectomy and ADAPT. J Neurointerv Surg 8 : 197202, 2016

4. Fargen KM, Arthur AS, Spiotta AM, Lena J, Chaudry I, Turner RD, et al. : A survey of neurointerventionalists on thrombectomy practices for emergent large vessel occlusions. J Neurointerv Surg 9 : 142-146, 2017

5. Goyal M, Demchuk AM, Menon BK, Eesa M, Rempel JL, Thornton J, et al. : Randomized assessment of rapid endovascular treatment of ischemic stroke. N Engl J Med 372 : 1019-1030, 2015

6. Goyal M, Menon BK, van Zwam WH, Dippel DW, Mitchell PJ, Demchuk AM, et al. : Endovascular thrombectomy after large-vessel ischaemic stroke: a meta-analysis of individual patient data from five randomised trials. Lancet 387 : 1723-1731, 2016

7. Higashida RT, Furlan AJ, Roberts H, Tomsick T, Connors B, Barr J, et al. : Trial design and reporting standards for intra-arterial cerebral thrombolysis for acute ischemic stroke. Stroke 34 : e109-e137, 2003

8. Kang DH, Park J : Endovascular stroke therapy focused on stent retriever thrombectomy and direct clot aspiration: historical review and modern application. J Korean Neurosurg Soc 60 : 335-347, 2017

9. Kerr DM, Fulton RL, Lees KR; VISTA Collaborators : Seven-day NIHSS is a sensitive outcome measure for exploratory clinical trials in acute stroke: evidence from the Virtual International Stroke Trials Archive. Stroke 43 : 1401-1403, 2012

10. Kleine JF, Wunderlich S, Zimmer C, Kaesmacher J : Time to redefine success? $\mathrm{TICl} 3$ versus $\mathrm{TICI} 2 \mathrm{~b}$ recanalization in middle cerebral artery occlusion treated with thrombectomy. J Neurointerv Surg 9 : 117-121, 2017

11. Linfante I, Starosciak AK, Walker GR, Dabus G, Castonguay AC, Gupta $R$, et al. : Predictors of poor outcome despite recanalization: a multiple regression analysis of the NASA registry. J Neurointerv Surg 8 : 224229, 2016

12. Mokin M, lonita CN, Nagesh SV, Rudin S, Levy El, Siddiqui AH : Primary stentriever versus combined stentriever plus aspiration thrombectomy approaches: in vitro stroke model comparison. J Neurointerv Surg 7 : 453-457, 2015

13. Mueller-Kronast NH, Zaidat 00, Froehler MT, Jahan R, Aziz-Sultan MA, Klucznik RP, et al. : Systematic evaluation of patients treated with neurothrombectomy devices for acute ischemic stroke: primary results of the STRATIS registry. Stroke 48 : 2760-2768, 2017

14. Nguyen TN, Malisch T, Castonguay AC, Gupta R, Sun CH, Martin CO, et al. : Balloon guide catheter improves revascularization and clinical outcomes with the solitaire device: analysis of the North American Solitaire Acute Stroke Registry. Stroke 45 : 141-145, 2014

15. Oh JS, Yoon SM, Shim JJ, Doh JW, Bae HG, Lee KS : Efficacy of balloonguiding catheter for mechanical thrombectomy in patients with anterior circulation ischemic stroke. J Korean Neurosurg Soc 60 : 155-164, 2017

16. Powers WJ, Derdeyn CP, Biller J, Coffey CS, Hoh BL, Jauch EC, et al. : 2015 American Heart Association/American Stroke Association focused update of the 2013 guidelines for the early management of patients with acute ischemic stroke regarding endovascular treatment: a guideline for healthcare professionals from the American Heart Association/American Stroke Association. Stroke 46 : 3020-3035, 2015

17. Velasco A, Buerke B, Stracke CP, Berkemeyer S, Mosimann PJ, Schwindt $W$, et al. : Comparison of a balloon guide catheter and a non-balloon guide catheter for mechanical thrombectomy. Radiology 280 : 169176, 2016

18. Young FB, Weir CJ, Lees KR; GAIN International Trial Steering Committee and Investigators : Comparison of The National Institutes of Health Stroke Scale with disability outcome measures in acute stroke trials. Stroke 36 : 2187-2192, 2005 